\title{
A case of chronic unilateral maxillary sinusitis by Pseudoallescheria boydii
}

\author{
Jeevan Malaiyan ${ }^{1, *}$, Sowmya Nasimuddin ${ }^{1}$, Gokul Radhakrishnan ${ }^{3}$, \\ Sunitha Munirathinam ${ }^{2}$, Mohanakrishnan Kandasamy ${ }^{1}$, Ashokan L ${ }^{2}$, \\ Sambandan Alandur Paramasivan ${ }^{2, *}$ \\ 1Department of Microbiology, Sri Muthukumaran Medical College Hospital and Research Institute, \\ Chikkarayapuram, Chennai-600 069, India. \\ 2 Department of ENT, Sri Muthukumaran Medical College Hospital and Research Institute, \\ Chikkarayapuram, Chennai-600 069, India. \\ 3 Department of Medicine, Meenakshi Medical College Hospital and Research Institute, Enathur, \\ Kanchipuram-631 552, India.
}

\begin{abstract}
Pseudallescheria boydii an emerging pathogen of humans is a filamentous fungus frequently isolated from respiratory infections. It is commonly believed that infection by this fungus occurs through inhalation of airborne conidia, but the mechanisms allowing the adherence of Pseudallescheria to the host epithelial cells and its escape from the host immune defences remain largely unknown. This fungus causes a wide array of human infections that can affect practically all the organs of the body. Disseminated and invasive infections with this fungus are seen primarily in immunocompromised hosts. Here, we report a case of unilateral maxillary sinusitis caused by $P$. boydii in an immunocompetant male. The patient was surgically treated and was followed up with antifungal and is free of symptoms, with no recurrence after 6 months.
\end{abstract}

Keywords: antifungal, maxillary sinusitis, Pseudallescheria boydii, recurrence.

\section{Introduction:}

Pseudoallescheria boydii ( $P$. boydii), belonging to the group Hyalohyphomycosis, is frequently isolated from soil, manure and decaying vegetation, having a worldwide distribution [1]. P. boydii has long been known to cause white grain mycetoma in immunocompetent humans, but it has recently emerged as an opportunistic pathogen of humans, causing potentially fatal invasive infections in immunocompromised individuals [2]. The mechanisms of adherence and establishment of an infection by these fungi in the lung are still largely unknown. It is thought that the infection process in the respiratory tract starts by inhalation and adhesion of airborne conidia that differentiate into hyphae, with both processes mediated by the spore cell wall since that acts as the interface between the fungus and lung tissues [3]. Aspergillus fumigatus is the most common species implicated in paranasal sinus infection, with other species being rarely reported. Like Aspergillus spp, P. boydii can colonize pre-existing cavities. Though less invasive than Mucoracea and Aspergillus, $P$. boydii can be invasive in severely immunosuppressed individuals [4]. Here we report a case of chronic unilateral fungal maxillary sinusitis caused by P.boydii.

\section{Case history:}

A 48 year old male patient, who is carpenter by occupation, presented to the hospital with a history of chronic headache, right sided nasal block and nasal discharge for a period of six months. No significant past history was reported. On examination, a deviated nasal septum to the right was observed. CT scan showed a polypoidal expansile soft tissue density in right maxillary sinus, extending into right nasal cavity, causing widening of the ostium, with mild mucosal thickening in the bilateral frontal, ethmoidal and sphenoidal sinuses. On diagnostic nasal endoscopy, polypoidal mass was observed in right nasal cavity (Figure 1). Functional endoscopic sinus surgery was done and a grayish black mass was removed from right maxillary sinus. Left maxillary sinus, bilateral ethmoidal, sphenoidal and frontal sinuses were normal. On microscopic examination with potassium hydroxide mount $(\mathrm{KOH})$, fungal elements were seen. Fungal culture was done in Sabouraud's dextrose agar (SDA) and was incubated at $37^{\circ} \mathrm{Cand} 24^{\circ} \mathrm{C}$. After 48 hours of incubation, white cottony fluffy colonies appeared in culture at $37^{\circ} \mathrm{C}$, with reverse being greyish white in colour (Figure 2). Microscopically, septate hyphae with conidiophores and oval conidia borne singly from tips were observed (Figure 3). On histopathological examination, septate hyphae were found with granulomatous inflammation. The fungal isolate was identified as $P$. boydii from its characteristic microscopic and macroscopic morphology. 


\section{Discussion:}

P. boydii is a ubiquitous ascomycetous fungus that causes a wide array of human infections that can affect practically all the organs of the body. These infections have been known for a long time, but in recent years, a marked increase in severe invasive infections has been noticed, mainly in immunocompromised hosts. The treatment of these infections has not yet been resolved, and the mortality rate is very high and main portal of entry being the respiratory tract and with possible dissemination to other target organs [5]. Among the paranasal sinuses, the commonest site involved is the maxillary sinus, followed by sphenoidal sinus. In our case report, the patient presented with chronic unilateral maxillary sinusitis, which was diagnosed to be fungal sinusitis caused by $P$. boydii. The diagnosis was confirmed by laboratory findings. The patient was subsequently treated medically and surgically and responded well. Very few cases of fungal sinusitis caused by $P$. boydii have been reported in literature in immunocompetant individuals [6]. In conclusion, fungal sinusitis secondary to $P$. boydii infection is rare. In general, patients should be treated with a combination of surgery and antifungal therapy, particularly those patients with immunosuppression and fungal invasion. Immunocompetent patients with $P$. boydii fungal sinusitis have a better prognosis, even when invasion is present.

\section{References:}

[1]. Rippon JW. Pseudallescheriasis. In: Wonsiewicz M, ed. Medical Mycology: The Pathogenic Fungi and the Pathogenic Actinomycetes. 3rd ed. Philadelphia: W.B. Saunders: 1988: 651-3.

[2]. Thornton CR. Tracking the Emerging Human Pathogen Pseudallescheria boydii by Using Highly Specific Monoclonal Antibodies. Clinical and Vaccine Immunology 2009; 16(5): 756-764.

[3]. Ghamrawi S, Rénier G, Saulnier P, Cuenot S, Zykwinska A, Dutilh BE, Thornton C, et al. Cell Wall Modifications during Conidial Maturation of the Human Pathogenic Fungus Pseudallescheria boydii. Plos One 2014; 20; 9(6): e100290.

[4]. Chavan SS, Makannavar JH. Chronic sino-naso-orbital fungal infection due to Pseudallescheria boydii infection in a nonimmunocompromised host: A case report. Indian J Pathol Microbiol 2001; 44(3): 59-61.

[5]. Gilgado F, Cano J, Gene J, Guarro J. Molecular phylogeny of the Pseudallescheria boydii species complex: proposal of two species. J Clin Microbiol. 2005; 43(10): 4930-42.

[6]. Winn RE, Ramsey PD, McDonald JC, Dunlop KJ. Maxillary sinusitis from Pseudallescheria boydii. Efficacy of surgical therapy. Arch Otolaryngol. 1983; 109(2):123-5.

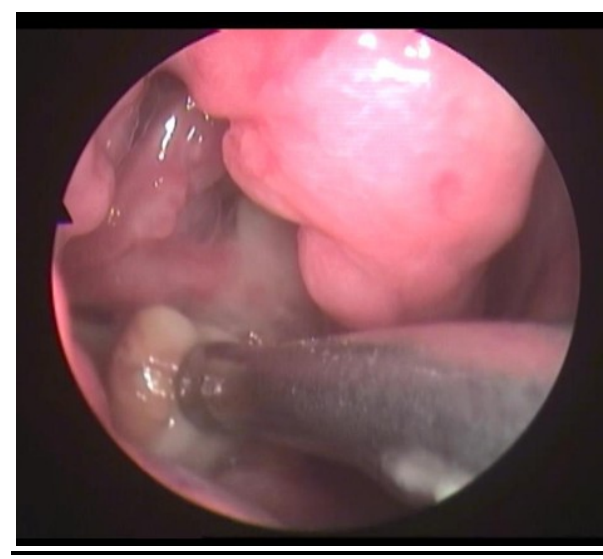

Figure 1: Nasal endoscopy: local examination of the nasal cavity on right side reveals a polypoidal mass. 


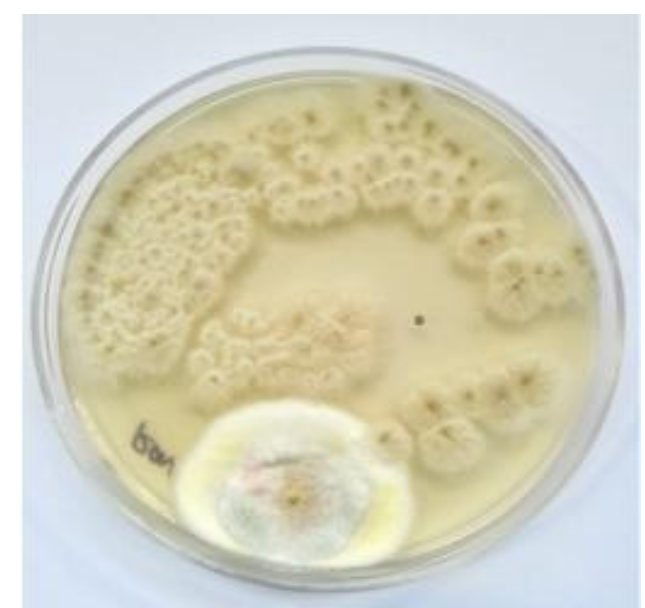

Figure 2: Fungal culture: White cottony feathery colonies on SDA.

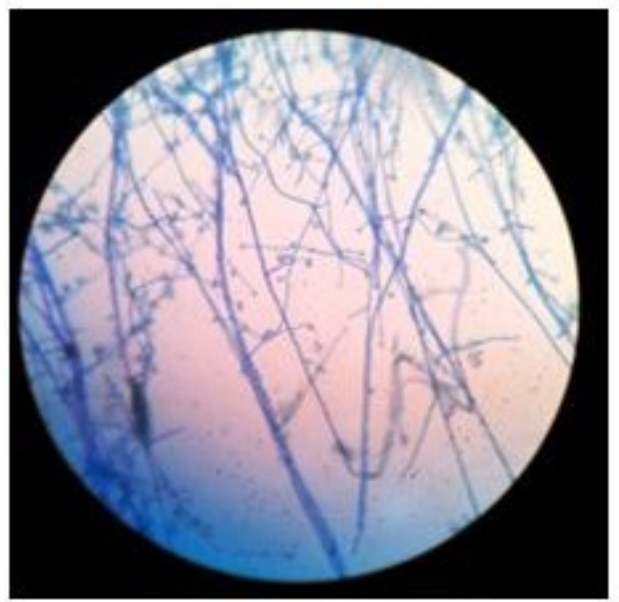

Figure 3: LPCB mount: Septate hyphae with conidiophore and oval conidia Pseudallescheria boydii. 